\title{
Cuidados de enfermagem para prevenção e manejo da Hipertensão Arterial em
}

\section{gestantes na Atenção Primária}

\author{
Nursing care for the prevention and management of hypertension in pregnant women in Primary \\ Care
}

Cuidados de enfermería para la prevención y el manejo de la hipertensión en mujeres embarazadas en Atención Primaria

\author{
Débora Thaís Rodrigues de Sousa \\ ORCID: https://orcid.org/0000-0002-2795-5195 \\ Centro Universitário Santo Agostinho, Brasil \\ E-mail: deboraa.thais18@gmail.com \\ Estefany de Jesus Silva \\ ORCID: https://orcid.org/0000-0001-8849-759X \\ Centro Universitário Santo Agostinho, Brasil \\ E-mail estafanyjc19@gmail.com \\ Raquel Vilanova Araújo \\ ORCID: https://orcid.org/0000-0001-5902-9869 \\ Centro Universitário Santo Agostinho, Brasil \\ E-mail: raquelvila@outlook.com
}

\begin{abstract}
Resumo
O objetivo do estudo é identificar os cuidados de enfermagem para prevenção e manejo da Hipertensão Arterial em gestantes na atenção primária. Revisão integrativa. A busca foi realizada nas bases de dados LILACS, MEDLINE, SCIELO, via portal de periódicos capes com os Mesh "Hypertension, pregnancy-induced" OR "prenatal care" OR "primary care nursing". Na amostra inicial tem-se um total de 52.595 artigos, após utilização dos critérios de inclusão e exclusão, obteve-se um total de 39.997 estudos e após a leitura dos títulos e resumos, obteve-se uma amostra final de 1.345 estudos que foram lidos com rigor e em sua íntegra, a amostra final foi composta de 16 estudos que foram analisados, interpretados e discutidos. Após análise das publicações constatou-se a importância da assistência de enfermagem no pré-natal, bem como da qualificação dos profissionais no manejo adequado das gestantes hipertensas e, portanto, na prevenção das possíveis complicações, que deve repercutir de maneira positiva na redução das taxas de mortalidade materno- infantil. Evidenciou-se no estudo o significativo percentual de mulheres jovens com hipertensão durante a gestação assim como a existência de fatores de risco entre estas mulheres. Faz-se necessário as intervenções de enfermagem envolvendo o controle da hipertensão arterial, acolhimento, suporte emocional e espiritual para as gestantes e seus familiares, e no desenvolvimento de estratégias que possam contribuir para a redução da ansiedade e estresse, e incentivo à prática de atividade física, orientação quanto o uso das medicações, controle e acompanhamento do peso corporal e controle nutricional.
\end{abstract}

Palavras-chave: Hipertensão induzida pela gravidez; Cuidados de enfermagem; Enfermagem de atenção primária.

\begin{abstract}
The aim of the study is to identify nursing care for the prevention and management of hypertension in pregnant women in primary care. Integrative literature review. The search was conducted in the LILACS, MEDLINE, SCIELO databases, via the Capes periodicals portal with the Mesh "Hypertension, pregnancy-induced" OR "prenatal care" OR "primary care nursing". The initial sample contained a total of 52,595 articles. After using the inclusion and exclusion criteria, a total of 39,997 studies were obtained and after reading the titles and abstracts, a final sample of 1,345 studies was obtained. After analyzing the publications, it was observed the importance of nursing care during prenatal care, as well as the qualification of professionals in the appropriate management of hypertensive pregnant women and, therefore, in the prevention of possible complications, which should have a positive impact on reducing maternal and infant mortality rates. The significant percentage of young women with hypertension during pregnancy, as well as the existence of risk factors among these women, was evident in the study. It is necessary the nursing interventions involving the control of hypertension, reception, emotional and spiritual upport for pregnant women and their families, and the development of strategies that can contribute to reducing anxiety and stress, and encouragement of physical activity, guidance on the use of medications, control and monitoring of body weight and nutritional control.
\end{abstract}

Keywords: Pregnancy-induced hypertension; Nursing care; Primary care nursing. 


\section{Resumen}

El objetivo del estudio es identificar los cuidados de enfermería para la prevención y el manejo de la hipertensión en mujeres embarazadas en atención primaria. Revisión bibliográfica integradora. La búsqueda se realizó en las bases de datos LILACS, MEDLINE, SCIELO, a través del portal de publicaciones periódicas Capes con la malla "Hypertension, pregnancy-induced" OR "prenatal care" OR "primary care nursing". Tras utilizar los criterios de inclusión y exclusión, se obtuvo un total de 39.997 estudios y, tras leer los títulos y resúmenes, se obtuvo una muestra final de 1.345 estudios. Tras el análisis de las publicaciones, se constató la importancia de los cuidados prenatales de enfermería, así como la cualificación de los profesionales en el manejo adecuado de las gestantes hipertensas y, por tanto, en la prevención de posibles complicaciones, lo que debería repercutir positivamente en la reducción de las tasas de mortalidad maternoinfantil. El estudio puso de manifiesto el importante porcentaje de mujeres jóvenes con hipertensión durante el embarazo, así como la existencia de factores de riesgo entre estas mujeres. Son necesarias las intervenciones de enfermería que impliquen el control de la hipertensión, la acogida, el apoyo emocional y espiritual a la mujer embarazada y a su familia, y el desarrollo de estrategias que puedan contribuir a reducir la ansiedad y el estrés, así como el fomento de la actividad física, la orientación sobre el uso de medicamentos, el control y la vigilancia del peso corporal y el control nutricional.

Palabras clave: Hipertensión inducida por el embarazo; Cuidados de enfermería; Enfermería de atención primaria.

\section{Introdução}

A Hipertensão arterial sistêmica, é caracterizada pela pressão sistólica igual ou maior que $140 \mathrm{mmHg}$ e diastólica igual ou maior que $90 \mathrm{mmHg}$, com base na média de pelo menos duas medidas (Nascimento et al., 2017). Já a Hipertensão gestacional, é caracterizada quando há um aumento na pressão arterial sistólica superior a $30 \mathrm{mmHg}$ e/ou na pressão diastólica, superior a 15 $\mathrm{mmHg}$, pela primeira vez durante a gestação; e devido as intercorrências e possíveis complicações isso pode ocasionar danos à saúde da mãe e do feto (Gonçalves et al., 2019).

A gestação é um momento muito especial e esperado na vida das mulheres, no entanto durante esse período algumas patologias podem ser diagnosticadas como é o caso da hipertensão gestacional, que de acordo com Sousa et al (2019), é considerada um dos principais problemas de saúde pública, em decorrência das elevadas taxas de morbidade e mortalidade materna e perinatal, com um percentual alto de incidência no Brasil e no mundo. É importante salientar que, a mesma acomete gestantes de todas as idades, trazendo complicações e levando ao óbito.

Quando se desenvolve na gestação, a hipertensão pode estar relacionada a fatores genéticos, ambientais e imunológicos, como diabetes, obesidade, gravidez multípara, doença renal, primiparidade, idade superior a 30 anos, hipertensão crônica e/ou pré-eclâmpsia, antecedentes pessoais ou familiares, hábito alimentar inadequado e o sedentarismo (Araújo et al.,2017).

O número elevado de gestantes com hipertensão arterial e com vários fatores de risco associados, é de suma importância para uma avaliação completa da gestante a fim de identificar precocemente estes fatores de risco e adotar medidas preventivas e manejar a gestante de maneira adequada durante todo o pré-natal a fim de risco de complicações e intercorrências (Costa $e t$ al.,2016).

Destaca-se a importância das Políticas Públicas voltadas para o acesso as consultas de pré-natal, do planejamento familiar, identificação dos fatores de risco, início precoce e qualidade da assistência no pré-natal, manejo adequado, acolhimento e acompanhamento destas gestantes (Nóbrega et al., 2016). Segundo Pereira et al., (2017), as síndromes hipertensivas na gravidez são classificadas em pré-eclâmpsia, eclampsia, hipertensão crônica sobreposta à pré-eclâmpsia, hipertensão crônica e hipertensão gestacional.

A consulta de pré-natal é uma ferramenta essencial para a promoção e segurança materna e fetal, bem como para o acompanhamento da gestante, planejamento da assistência e desenvolvimento de ações com a finalidade de prevenir a hipertensão gestacional e as possíveis complicações, sendo essencial a classificação de risco a cada consulta. Destacando assim, a importância da consulta pré-concepcional para a identificação dos fatores de risco e das orientações quanto a mudança no estilo de vida a fim de evitar a ocorrência da hipertensão durante a gestação (Lima et al.,2018). 
O profissional de enfermagem, ao acompanhar a mulher durante a gestação, deve buscar condutas específicas individualizadas, manejar a gestante de maneira adequada, identificar precocemente as situações de risco e patologias e, portanto, prestar um atendimento de maneira eficaz e seguro, a fim de evitar complicações futuras (Mariano et al.,2018).

Diante da problemática, surge como questão norteadora do estudo: Quais os cuidados de enfermagem para prevenção e manejo da hipertensão arterial em gestantes? Assim o objetivo do estudo é identificar os cuidados de enfermagem para prevenção e manejo da hipertensão arterial sistêmica em gestantes.

\section{Metodologia}

Trata-se de uma revisão integrativa, pois é um método que permite a procura, a avaliação crítica e a síntese das evidências, em que o produto final é o estado do conhecimento do tema investigado, a implementação de intervenções efetivas na prestação de cuidados e na redução de custos, além disso permite a identificação de fragilidades, que poderão conduzir ao desenvolvimento de futuras investigações (Sousa et al., 2017).

Essa revisão foi estruturada em seis fases distintas: identificação do tema e seleção da questão de pesquisa para a elaboração da revisão integrativa; estabelecimento de critérios para inclusão e exclusão de estudos/amostragem ou pesquisa de literatura; definição das informações a serem extraídas dos estudos selecionados/categorização dos estudos; avaliação dos estudos incluídos na revisão integrativa; interpretação dos resultados e, apresentação da revisão/síntese do conhecimento (Mendes; Silveira \& Galvão, 2008).

A busca de dados foi realizada nas bases de dados da Literatura Latino-Americano em Ciências da Saúde (LILACS), Scientific Eletronic Libray Online (SCIELO), Sistema de Busca e Análise de Literatura Médica (MEDLINE), via portal de periódicos capes com o Mesh "Hypertension pregnancy- induced" OR "Prenatal care" OR "Primary cary nursing". A busca nas bases de dados ocorreu entre Fevereiro e Março de 2021. Considerou-se como critério de inclusão estudos primários publicados na língua portuguesa, inglesa e espanhola e que correspondiam a temática do estudo e publicados nos últimos 5 anos. Optou-se por utilizar o recorte temporal com a finalidade de apreciar as evidências e tendências mais anuais relacionadas a temática do estudo. E excluídos editoriais, teses, dissertações, monografias, manuais, estudos duplicados e estudos de revisão de literatura.

Para responder à pergunta norteadora de estudo "Quais os cuidados de enfermagem para a prevenção e manejo da Hipertensão Arterial em gestantes". Utilizou-se o acrômio PICo, onde P (População- gestantes com hipertensão); I (Interesseações de enfermagem na atenção primária); Co (Contexto-cuidado pré- natal) (Araújo,2020). A estratégia de busca encontra-se descrita no quadro abaixo. 
Research, Society and Development, v. 10, n. 6, e1410615464, 2021

(CC BY 4.0) | ISSN 2525-3409 | DOI: http://dx.doi.org/10.33448/rsd-v10i6.15464

Quadro 1 - Estratégia de busca segundo acrômio PICo.

\begin{tabular}{|c|c|c|c|}
\hline $\begin{array}{l}\text { Objetivo/ } \\
\text { Problema }\end{array}$ & \multicolumn{2}{|c|}{ Quais os cuidados de enfermagem para a prevenção e manejo da hipertensão arterial em gestantes? } \\
\hline & P & I \\
\hline Decs & Hipertensão Induzida pela Gravidez & Cuidados de Enfermagem & Enfermagem de Atenção Primária \\
\hline Mesh & Hypertension, Pregnancy-Induced & Primary Care Nursing \\
\hline LILACS & "Hypertension, pregnancy-induced" OR "nursing care" OR "primary care nursing" \\
\hline SCIELO & (Hypertension, Pregnancy-Induced) OR (Nursing Care) OR (Primary Care Nursing) \\
\hline MDLINE & Hypertension, pregnancy-induced OR Primary care nursing OR prenatal care. \\
\hline
\end{tabular}

Fonte: BVS.

Os dados dos estudos selecionados foram coletados com o auxílio de um ficha de extração de dados com informações relacionadas ao título do artigo, ano de publicação, tipo de estudo, abordagem metodológica, objetivos, ações de enfermagem para a prevenção e manejo da hipertensão em gestantes durante o pré-natal.

Os dados coletados foram organizados e descritos em quadros, tabelas e gráficos, e calculados utilizando-se uma porcentagem simples por meio da ferramenta estatística no Microsoft Office Excel.

\section{Resultados}

Foram recuperados 52.595 estudos, após utilização dos critérios de inclusão e exclusão, obteve-se um total de 39.997 estudos e após a leitura dos títulos e resumos, obteve-se uma amostra final de 1. 345 estudos que foram lidos com rigor e em sua íntegra, a amostra final foi composta de 16 estudos que foram analisados, interpretados e discutidos. Nas etapas da seleção das publicações foram consideradas as recomendações do Preferred Reporting Items for Systematic Reviews and Meta-Analyses (PRISMA) (Moher et al., 2009), que encontra-se na Figura 1. 
Figura 1 - Fluxograma PRISMA representativo do processo de Revisão de Literatura. Teresina- PI, 2021.
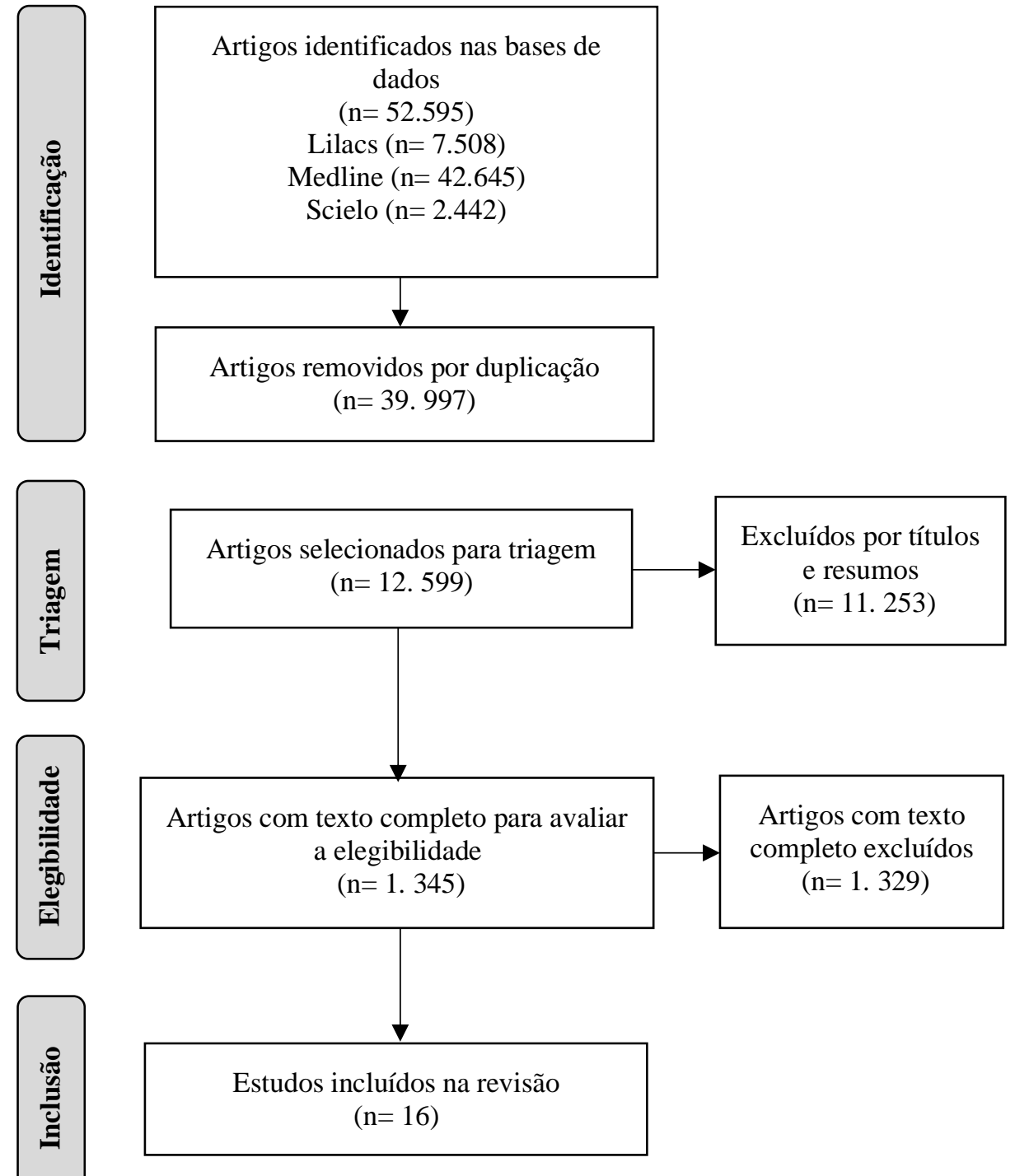

Fonte: Dados da pesquisa (2021).

$\mathrm{Na}$ Tabela 1 verifica-se que de acordo com as evidências cientificas encontradas o maior percentual foi de pesquisas com abordagem quantitativa (75\%), do tipo descritiva (25\%) e desenvolvidas em São Paulo (31,25\%). 
Tabela 1 - Distribuição em números absolutos e percentuais dos artigos segundo abordagem, tipo de estudo e local de estudo, Teresina/PI, 2021.

\begin{tabular}{lcc}
\hline \multicolumn{1}{c}{ Variáveis } & N & \% \\
\hline Tipo de abordagem & 12 & 75 \\
Quantitativa & 4 & 25 \\
Qualitativa & & \\
Tipo de Estudo & 2 & 12,5 \\
Observacional & 4 & 25 \\
Descritivo & 3 & 18,75 \\
Caso Controle & 3 & 18,75 \\
Estudo de Corte & 4 & 25 \\
Outros & & \\
Local de Estudo & 2 & 12,5 \\
Minas Gerais & 2 & 12,5 \\
Rio de Janeiro & 5 & 31,25 \\
São Paulo & 7 & 43,75 \\
Outros & & \\
\hline
\end{tabular}

Fonte: Dados da pesquisa (2021).

O Quadro 2, tem-se a caracterização das produções científicas, segundo nome dos autores, ano de publicação, título e objetivo.

Quadro 2 - Caracterização dos artigos conforme nome do autor e ano de publicação, título do estudo. Teresina/ PI, 2021.

\begin{tabular}{|c|c|c|}
\hline Autores/ Ano & Título & Objetivo do Estudo \\
\hline Assis et al., 2008. & $\begin{array}{l}\text { Estudo dos Principais Fatores de Risco Maternos } \\
\text { nas Síndromes Hipertensivas da Gestação. }\end{array}$ & Investigar os fatores de risco maternos para SHG. \\
\hline Soares et al.,2009. & $\begin{array}{c}\text { Mortalidade materna por pré- } \\
\text { eclâmpsia/eclampsia em um estado do Sul do } \\
\text { Brasil. }\end{array}$ & $\begin{array}{l}\text { Identificar o perfil, a tendência e os determinantes } \\
\text { da mortalidade materna por pré-eclâmpsia/ } \\
\text { Eclâmpsia no estado do Paraná. }\end{array}$ \\
\hline Coutinho et al.,2010. & $\begin{array}{c}\text { Monitoramento do processo de assistência pré-natal } \\
\text { entre as usuárias do Sistema Único de Saúde em } \\
\text { município do Sudeste brasileiro. }\end{array}$ & $\begin{array}{l}\text { Avaliar a evolução da adequação do processo de } \\
\text { atendimento às gestantes usuárias do Sistema Único de } \\
\text { Saúde (SUS) e consolidar metodologia para } \\
\text { monitoramento da assistência pré-natal. }\end{array}$ \\
\hline Silva et al., 2010. & $\begin{array}{c}\text { Avaliação das condutas de prevenção da síndrome } \\
\text { hipertensiva específica da gravidez entre } \\
\text { adolescentes. }\end{array}$ & $\begin{array}{l}\text { Avaliar as condutas em } \\
\text { adolescentes grávidas na prevenção e/ou controle dos } \\
\text { fatores de riscos da SHEG, com enfoque na educação } \\
\text { em saúde. }\end{array}$ \\
\hline $\begin{array}{l}\text { Novo \& Gianini, } \\
\quad 2010 .\end{array}$ & Mortalidade materna por Eclâmpsia. & $\begin{array}{l}\text { Analisar fatores associados à mortalidade materna } \\
\text { causada por Eclâmpsia. }\end{array}$ \\
\hline Vettore et al.,2011. & $\begin{array}{l}\text { Cuidados pré-natais e avaliação do manejo da } \\
\text { hipertensão arterial em gestantes do SUS no } \\
\text { Município do Rio de Janeiro, Brasil. }\end{array}$ & $\begin{array}{l}\text { Avaliar a adequação } \\
\text { atendimento pré-natal para gestantes com hipertensão } \\
\text { em comparação com aqueles de baixo risco. }\end{array}$ \\
\hline
\end{tabular}




\begin{tabular}{|c|c|c|}
\hline Nagle et al., 2011. & $\begin{array}{l}\text { Continuidade dos cuidados obstétricos gestacional } \\
\text { ganho de peso em mulheres obesas: um estudo } \\
\text { randomizado ensaio controlado. }\end{array}$ & $\begin{array}{l}\text { Avaliar o efeito da continuidade de } \\
\text { cuidados obstétricos para restringir o ganho de peso } \\
\text { gestacional em mulheres obesas ao intervalo } \\
\text { recomendado. }\end{array}$ \\
\hline Neto et al., 2013. & $\begin{array}{l}\text { Concordância entre informações do Cartão da } \\
\text { Gestante e do recordatório materno entre puérperas } \\
\text { de uma cidade brasileira de médio porte. }\end{array}$ & $\begin{array}{l}\text { Verificar a concordância entre as informações dadas } \\
\text { pelas mães após entrega e dados registrados nos } \\
\text { cartões de gestante sobre o pré-natal no âmbito do } \\
\text { Unificado Brasileiro Sistema Único de Saúde da } \\
\text { Região Metropolitana de Vitória, Espírito Santo, } \\
\text { Brasil. }\end{array}$ \\
\hline Spindola et al., 2013. & $\begin{array}{l}\text { A ocorrência de pré-eclâmpsia em mulheres } \\
\text { primigestas acompanhadas no pré-natal de um } \\
\text { hospital universitário. }\end{array}$ & $\begin{array}{l}\text { Conhecer o perfil das gestantes primigestas atendidas } \\
\text { no pré-natal; identificar a incidência de pré-eclâmpsia } \\
\text { em gestantes primigestas atendidas em consultas do } \\
\text { pré-natal. }\end{array}$ \\
\hline González et al, 2016. & $\begin{array}{l}\text { Resultados perinatales en gestantes con trastornos } \\
\text { hipertensivos del embarazo, } \\
\text { hospital regional Santa Teresa, } 2015 .\end{array}$ & $\begin{array}{l}\text { Descrever as características clínicas e epidemiológicas } \\
\text { dos recém-nascidos de mães com distúrbios } \\
\text { hipertensivos da gravidez na sala de trabalho de parto } \\
\text { e parto do Hospital Regional Santa Teresa, } \\
\text { Comayagua, durante } 2015 \text {. }\end{array}$ \\
\hline Tuon et al., 2016. & $\begin{array}{l}\text { Impacto do monitoramento telefônico de gestantes na } \\
\text { prevalência da prematuridade } \\
\text { e análise dos fatores de risco associados em } \\
\text { Piracicaba, São Paulo, Brasil. }\end{array}$ & $\begin{array}{l}\text { Verificar o impacto do monitoramento telefônico na } \\
\text { prevalência da prematuridade e identificar os fatores } \\
\text { de risco associados ao parto prematuro. }\end{array}$ \\
\hline Silva et al., 2017. & $\begin{array}{c}\text { Cuidados pré-natais e puerperais às gestantes de um } \\
\text { centro de saúde de Minas Gerais quanto ao risco de } \\
\text { Pré-eclâmpsia: aspectos clínicos, nutricionais e } \\
\text { terapêuticos }\end{array}$ & $\begin{array}{l}\text { Avaliar os aspectos clínicos, nutricionais e } \\
\text { terapêuticos nos cuidados pré-natais e puerperais às } \\
\text { gestantes de um centro de saúde de Minas Gerais } \\
\text { quanto ao risco de Pré-eclâmpsia. }\end{array}$ \\
\hline Sampaio et al.,2018. & $\begin{array}{l}\text { Gestação de alto risco: perfil clínico-epidemiológico } \\
\text { das gestantes atendidas no serviço de pré-natal da } \\
\text { maternidade pública de Rio Branco, Acre. }\end{array}$ & $\begin{array}{l}\text { Descrever o perfil clínico e epidemiológico das } \\
\text { gestantes atendidas no serviço de pré-natal de alto } \\
\text { risco da Maternidade Pública de Rio Branco, Acre. }\end{array}$ \\
\hline Peraçoli et al.,2019. & Pré-eclâmpsia/Eclampsia & $\begin{array}{l}\text { Explora a etiologia ainda desconhecida, aspectos } \\
\text { atuais da fisiopatologia e do diagnóstico e diagnóstico } \\
\text { diferencial de convulsões, a abordagem da predição da } \\
\text { doença, seus resultados adversos e prevenção. }\end{array}$ \\
\hline Rezende et al., 2020. & $\begin{array}{l}\text { Resultados maternos e perinatais de gestações } \\
\text { complicadas por hipertensão crônica acompanhada } \\
\text { em um hospital de referência. }\end{array}$ & $\begin{array}{l}\text { Avaliar os resultados maternos e perinatais em } \\
\text { gestação de mulheres com hipertensão crônica. }\end{array}$ \\
\hline Lopes et al.,2020. & Epidemiologia da hipertensão arterial em gestantes. & $\begin{array}{l}\text { Pesquisar os dados epidemiológicos da hipertensão } \\
\text { arterial em gestantes, bem como identificar seus } \\
\text { possíveis eventos associados. }\end{array}$ \\
\hline
\end{tabular}


Na Tabela 2, de acordo com as evidências encontradas observa-se que é maior o percentual de gestantes é mulheres jovens $(62,5 \%)$, com ensino médio completo $(18,75 \%)$, casadas $(56,25 \%)$, primíparas $(37,5 \%)$, com hipertensão crônica instalada $(37,5 \%)$, e outros fatores de risco como sobrepeso, obesidade, sedentarismo $(18,75 \%)$, respectivamente.

Tabela 2 - Perfil das gestantes diagnosticadas com Hipertensão Arterial durante o pré-natal. Teresina/PI, 2021.

\begin{tabular}{|c|c|c|}
\hline Variáveis & $\mathbf{N}$ & $\%$ \\
\hline \multicolumn{3}{|l|}{ Faixa Etária (anos) } \\
\hline $15-29$ & 10 & 62,5 \\
\hline$>30$ & 6 & 37,5 \\
\hline \multicolumn{3}{|l|}{ Escolaridade } \\
\hline Sem escolaridade & 0 & 0 \\
\hline Ensino fundamental completo & 3 & 18,75 \\
\hline Ensino médio completo & 9 & 56,25 \\
\hline Ensino superior & 4 & 25 \\
\hline \multicolumn{3}{|l|}{ Situação conjugal } \\
\hline Solteira & 5 & 31,25 \\
\hline Casada & 9 & 56,25 \\
\hline Divorciada/ viúva & 2 & 12,5 \\
\hline \multicolumn{3}{|c|}{ Renda familiar (Salários mínimos) } \\
\hline Abaixo de 01 & 5 & 31,25 \\
\hline 01 & 10 & 62,5 \\
\hline $2-3$ & 1 & 6,25 \\
\hline \multicolumn{3}{|l|}{ Gravidez } \\
\hline Primípara & 10 & 62,5 \\
\hline Multípara & 6 & 37,5 \\
\hline \multicolumn{3}{|l|}{ Fatores de risco } \\
\hline Hipertensão arterial crônica & 6 & 37,5 \\
\hline Sedentarismo & 3 & 18,75 \\
\hline Sobrepeso/ Obesidade & 3 & 18,75 \\
\hline Gestação gemelar & 3 & 18,75 \\
\hline Diabetes mellitus & 1 & 6,25 \\
\hline
\end{tabular}

Fonte: Dados da pesquisa (2021).

No Gráfico 1 tem-se as principais ações de enfermagem para a prevenção da prevenção e manejo da Hipertensão Arterial durante o pré-natal, de acordo com as evidências científicas encontradas, observa-se que foi mais frequente as orientações relacionadas ao controle do peso e alimentação balanceada. 
Gráfico 1 - Ações de Enfermagem para prevenção e manejo da Hipertensão Arterial durante o pré-natal.

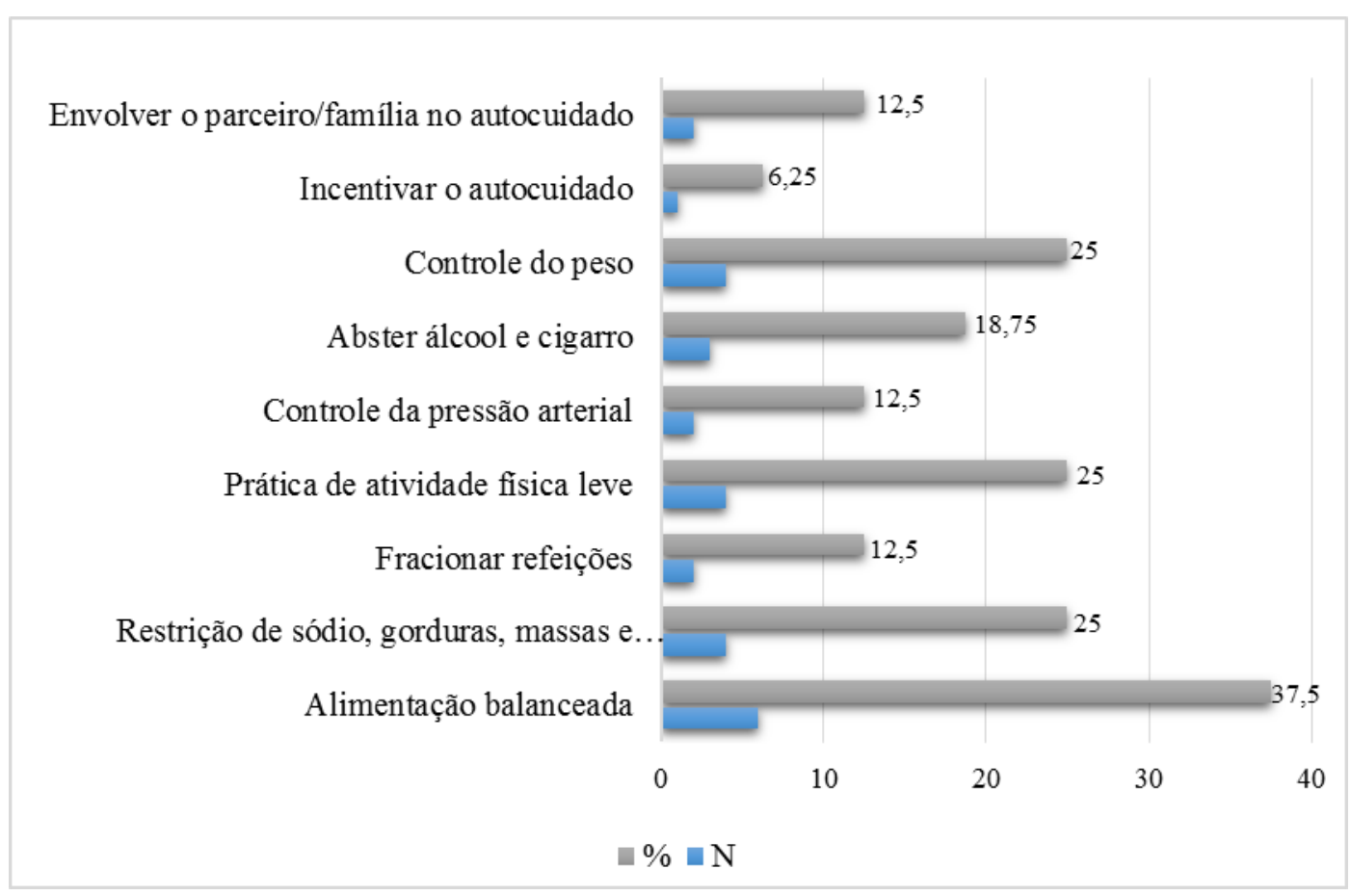

Fonte: Dados da pesquisa (2021).

No Quadro 3 observa-se os principais cuidados de enfermagem para o manejo adequado das gestantes com Hipertensão Arterial de acordo com as evidências científicas.

Quadro 3 - Cuidados de Enfermagem para o manejo adequado das gestantes Hipertensas durante o pré-natal.

- Realizar no mínimo as seis Consulta pré-natal;

- Realizar a classificação de risco da gestante em todas as consultas;

- Identificar e avaliar os fatores de risco para hipertensão, patologias de base e outros problemas;

- Realizar o exame físico completo da gestante;

- Verificar a pressão arterial e frequência cardíaca, mensuração do peso e altura, cálculo de IMC, avaliação de edemas, ausculta cardiopulmonar, altura uterina, idade gestacional, batimentos caridofetais, apresentação fetal, vitalidade fetal;

- Solicitar os exames de rotina do pré-natal;

- Analisar e interpretar o resultado dos exames solicitados (exames complementares, ginecológicos e ultrassonografia obstétrica);

- Encaminhar gestantes hipertensas para controle ambulatorial da pressão arterial semanal, se necessário;

- Encaminhar gestante hipertensa para o pré-natal de alto risco, quando necessário;

- Prevenir complicações relacionadas a hipertensão como pré-eclâmpsia e eclampsia;

- Orientar quanto o risco hipertensão para a mãe e para o feto;

- Orientar quanto o uso regular das medicações Anti-hipertensivas; 


\section{Discussão}

Reuniu-se nesta revisão evidências científicas sobre o perfil das gestantes com hipertensão arterial durante a gestação, as ações de enfermagem para a prevenção e os cuidados no manejo de gestantes com hipertensão arterial. Em relação ao perfil das gestantes com hipertensão na gestação através da análise das variáveis de interesse do estudo encontrou-se os seguintes dados: a prevalência gestantes tinham idade entre 15 a 29 anos com percentual $(62,5 \%)$ e maiores de 30 anos com percentual (37,5\%); sendo a maioria primíparas $(62,5 \%)$; casadas $(56,25 \%)$,e prevaleceu $(56,25 \%)$ das gestantes que concluíram o ensino médio, como fatores de risco a hipertensão arterial crônica (37,5\%), diabetes mellitus (6,25\%), e com sobrepeso/obesidade e gestação gemelar (18,75\%). O resultado encontrado é semelhante aquele observado em estudo realizado por Morais Neto et al (2018), onde verificou que a maioria das gestantes que participaram do estudo predominou mulheres jovens, que estavam na primeira gestação, com gestação gemelar e doenças pré-existentes como hipertensão arterial crônica e diabetes mellitus.

$\mathrm{Na}$ literatura observa-se que a respeito das ações para prevenção e manejo da hipertensão na gravidez, tem-se como uma das principais orientações, os cuidados com a alimentação e o incentivo à adoção de hábitos alimentares saudáveis (SOLBIATE et al, 2018). Segundo Almeida e Sousa (2016), as gestantes devem ser estimuladas e orientadas sobre o controle da ingestão de sódio na alimentação e incentivadas a incluir alimentos ricos em potássio na alimentação diária e fracionar as refeições durante o dia de forma quantitativa para uma dieta equilibrada.

Outras condutas têm sido indicadas para a prevenção da hipertensão gestacional, dentre estas a prática regular de atividade física. Segundo Martins et al (2016), a atividade física pode ser uma ação adotada durante o período gestacional e tem apresentado como benefícios para a gestante a diminuição de sintomas gravídicos, o melhor controle ponderal, manutenção da aptidão física e da saúde, auxílio no retorno venoso e melhora nas condições de irrigação da placenta. No entanto Gasparin et al (2018) ressalta que a prática de atividade física por gestantes deve ser acompanhadas e monitoradas por um profissional e que estas não causa qualquer tipo de prejuízo para a saúde materno-fetal, com exceção as gestantes com a patologia instalada e/ou situação que contra indique a realização da atividade física.

Segundo Fassarela et al (2020), os cuidados de enfermagem a serem prestados no que diz respeito ao manejo da hipertensão em gestantes são inúmeros, dentre estes destacam-se a aferição da pressão arterial em todas as consultas de pré-natal, controle da hipertensão crônica e a redução de condições estressantes e as orientações sobre os malefícios do consumo do álcool e do cigarro durante a gravidez. Corroborando com os achados encontrados, Thuler et al (2018), enfatiza a importância destas estratégias serem realizadas ainda no período de preconcepção já que a exposição a estas substâncias durante a gestação são prejudiciais tanto para mãe quanto para o feto.

A respeito da avaliação dos edemas, Abrahão et al (2020), explicam que esta deve ser realizado em todas as gestantes já que este pode ser caracterizado pelo aumento do peso corporal da gestante, tendo como consequência a hipertensão. Ademais, Kerber et al (2017) esclarece que quanto maior o IMC da gestante, maior a frequência dos casos de hipertensão arterial na gestação. Deste, diante deste problemática, destaca-se a importância do profissional de enfermagem incentivar as gestantes quanto o autocuidado, bem como a importância da prática de atividade física regular e controle do peso, a fim de prevenir o sobrepeso e obesidade, fatores predisponentes para a hipertensão arterial (Abrahão et al., 2020).

No estudo realizado verificou-se que é comum as gestantes juntamente com sua família/parceiro ir à unidade básica de saúde para a busca de suporte e orientações para compreender da melhor forma o momento que estão vivenciando, nesse contexto o enfermeiro aproveita para incentivar as gestantes sobre o autocuidado, fazendo com que elas se sintam mais tranquilas e seguras em relação ao período gestacional. Rocha et al (2016) explica que é fundamental dar suporte emocional a gestante bem como familiares, parceiro e acompanhantes.

Conforme análise dos estudos utilizado na pesquisa atual, verificou-se que a atenção e o acompanhamento da gestante nas consultas de pré-natal prestada de maneira humanizada e qualificada tem contribuído com a promoção de saúde e prevenção 
de complicações. Santos et al (2018) explica que durante o acompanhamento da gestante nas consultas de pré-natal o profissional saúde deve ter habilidade para fazer a classificação de risco da gestante e identificar situações de risco e, portanto, prevenir as complicações relacionadas a hipertensão arterial durante a gestação.

Antunes et al (2017), completa explicando que a equipe de enfermagem tem papel fundamental no manejo das gestantes ao longo de toda a gestação, no que tange a verificação da pressão arterial, altura uterina, peso materno, idade gestacional, edemas, batimentos caridofetais, vitabilidade fetal, resultados dos exames de rotina e obstétricos, bem como as orientações quanto o risco da hipertensão para a mãe e feto.

Jacob et al (2018), explica que a pré-eclâmpsia, uma das complicações relacionadas a hipertensão que pode interferir no processo de evolução natural da gestação e maternidade, de modo a trazer repercussões negativas na vida do binômio mãefilho. Deste modo Silva et al (2017), reforçam que é de suma importância as intervenções realizadas nos níveis de atenção primária, secundária e terciária para prevenir a hipertensão na gravidez e na detecção dos sinais e sintomas precoce a fim de prevenir complicações futura para a mãe feto, ainda mais pelo fato da hipertensão ser uma das principais causas de mortalidade materna e perinatal, mostrando-se assim a necessidade de uma assistência humanizada e qualificada por partes dos profissionais da saúde.

Os cuidados de Enfermagem para o manejo adequado das gestantes hipertensas durante o pré-natal envolveram as orientações quanto a regularidade nas consultas de pré-natal, realização de exames, uso de medicações, controle da pressão arterial, identificação dos sinais de alerta e orientação quanto os serviços de referência. Deste modo a importância da assistência de enfermagem no pré-natal, da assistência qualificada no planejamento familiar e no pré-natal, bem da qualificação e expertise dos profissionais no manejo adequado das gestantes hipertensas e, portanto, na prevenção das possíveis complicações, que deve repercutir de maneira positiva na redução das taxas de mortalidade materno- infantil.

\section{Conclusão}

Observa-se que é significativo o percentual de mulheres jovens com hipertensão durante a gestação, assim como a existência de fatores de risco entre estas mulheres como do sobrepeso, obesidade, sedentarismo, consumo de bebida alcóolica e fumo. Além disto percebeu-se que a maioria das gestantes possuem um baixo nível de escolaridade e são multíparas. Das ações de Enfermagem desenvolvidas para a prevenção e manejo da Hipertensão Arterial durante o pré-natal tem-se o incentivo ao autocuidado e as orientações acerca das mudanças no estilo de vida, hábitos alimentares saudáveis e evitar o uso de bebidas alcoólicas e fumo.

A realização desta pesquisa possibilitou mostrar que é fundamental a presença do enfermeiro, a fim de prestar um cuidado integral à gestante hipertensa, e desenvolver ações de conscientização acerca do autocuidado, controle da pressão arterial, acompanhamento ambulatorial, realização de exames e consultas de rotina. Em relação as intervenções de enfermagem estas devem envolver o controle da hipertensão arterial, acolhimento, suporte emocional e espiritual para as gestantes e seus familiares, e no desenvolvimento de estratégias que possam contribuir para a redução da ansiedade e estresse, e incentivo à prática de atividade física, orientação quanto o uso das medicações, controle e acompanhamento do peso corporal e controle nutricional.

\section{Referências}

Abrahão, A. C. M., Santos, R. F. S., Viana, S. R. G., \& Viana, S. M. (2020). Atuação do enfermeiro a pacientes portadoras de Síndrome Hipertensiva Específica da Gestação. Rev. Cient. Esc. Estadual Saúde Pública Goiás "Candido Santiago”, 6 (1),51- 63.

Almeida, G. B. S. \& Sousa, M. C. M. (2016). O Conhecimento da Gestante sobre a Hipertensão na Gravidez. Rev. APS. 19 (3), $396-402$. 
Antunes, M. B., Demitto, M. O., Gravena, A. A. F., Padovani, C. \& Pelloso, S. M.(2017). Síndrome Hipertensiva e Resultados Perinatais em Gestação de Alto Risco. Reme Rev. Min. Enfermagem, 21,1-5.

Araújo, Wánderson Cássio Oliveira. (2020) Recuperação da informação em saúde: construção, modelos e estratégias. Convergências em Ciência da Informação, 3(2),100-134.

Araújo, I. F. M., Santos, P. A. \& Franklin, T. A.(2017). Síndromes Hipertensivas e fatores de risco associadas a gestação. Revista de Enfermagem,10 (11), 4254-4262.

Assis, T. R., Viana. F. P, \& Rassi, S. (2008). Estudo dos Principais Fatores de Risco Maternos nas Síndromes Hipertensivas da Gestação. Arq. Bras. Cardiol, 91(1), 11-17.

Costa, L. D., Cura, C. C., Perondi, A. R., França, V. F. \& Bortoloti, D. S. (2016). Perfil epidemiológico de gestantes de alto risco. Cogitare Enferm, 2 (21), 27.

Coutinho, T., Monteiro, M. F. G., Sayd, G. D., Teixeira, M. T. B., Coutinho, C. M. \& Coutinho. M. (2010). Monitoramento do processo de assistência pré-natal entre as usuárias do Sistema Único de Saúde em município do Sudeste brasileiro. Rev. Bras. Ginecol. Obstet,32 (11), 563-569.

Fassarela, B. P. A., Almeida, G., Teles, D. A., Ortiz, L. S., Silva, I. S., Neves, K. C., Costa, P. A. F. S., Ribeiro, W. A. \& Evangelista, D. S. (2020). Cuidados de Enfermagem Direcionados à Gestante Portadora de Doença Hipertensiva Específica da Gravidez. Research Society And Development, 9 (9),1-20.

Gasparin, V. A., Favero, D. C., Albrecht, C. C., Gregolin, K. R., Pitilin, E. B \& Silva, D. T. R.(2018). Atividade Física em Gestantes como Prevenção da Síndrome Hipertensiva Gestacional. Rev. Enferm UFPE Online, 12 (4), 1017-1026.

Gonçalves, G. A., Paes, L. B. O., Parro, M. C., Olivares, N. M., Gambarini, T., Casanova, M. D.A S. \& Neto, A. C. A. (2019). Aspectos Sociodemográfico, clínico-obstétrico e laboratorial na Síndrome Hipertensiva na gravidez. Cuidarte Enfermagem, 13 (1), 27-31.

González, A. I. I., Fajardo, J. J. C., Izcoa, A. R., Reyes, V. G. A., Rivera, G. A. V., Lizardo, J. P. C., Fernández, T. B \& Fernández, R. D. (2016). Resultados perinatales en gestantes con trastornos Hipertensivos del embarazo, Hospital Regional Santa Teresa. Rev. Med. Hondur, 84, (1), 13-17.

Jacob, L. M. S., Alverne, D. G. B. M., Figueira, M. C. S., Mafetoni, R. R., Pimenta, C. J, L. \& Shimo, A. K. K. (2018). Conhecimento de Gestantes Sobre a Síndrome Hipertensiva Gestacional. Revista Enfermagem Atual In Derme, 24 (86), 1-8.

Kerber, G. F. \& Melere, C. (2017). Prevalência de síndromes hipertensivas gestacionais em usuárias de um hospital no sul do Brasil. Revista Cuidarte, 8(3),18991906.

Lima, J. P., Veras, L. L. N., Pedrosa, E. K. F., Oliveira, G. S. C. \& Guedes, M. V. C. (2018). Perfil socioeconômico e clínico de gestantes com Síndrome Hipertensiva Gestacional. http://periodicos.ufc.br/rene/article/view/33813. Acesso em: 02 set. 2020

Mariano, M. S. B., Belarmino, A. C., Vasconcelos, J. M. S. Holanda, L. C. A., Siqueira, D. D. \& Ferreira J. A. R.(2018). Mulheres com Síndromes Hipertensivas. Revista de Enfermagem, 12(6), 1618-1624.

Martins, A. B. T., Silva, J. G. B., Ponte, G. F., Filho, L., Saintrain, M. V. L., Caldas, J. M. P., Andrade, J. R. C. \& Palá cio, J. S. F. (2016). Adesão da Gestante ao Exercício Físico para a Prevenção e/ou Controle do Risco da Síndrome Hipertensiva. Rev. Bras. Promoção Saúde, 29(0), 25-35.

Mendes, K. D. S., Silveira, R. C. C. P. \& Galvão, C. M. (2008). Revisão integrativa: método de pesquisa para a incorporação de evidências na saúde e na enfermagem. Texto \& contexto enfermagem, 17(4), 758-764.

Moher, D., Liberati, A., Tetzlaff, J. \& Altman, D. G.(2019). Preferred Reporting Items for systematic Reviews and meta-Analyses: the PRISMA statement. Bmj, $1-8$.

Morais, N. H. N., Soares, F. A. F., Anjos, S. V., Nascimento, E. F. \& Santos, J. P. (2018). Fatores relacionados a ocorrência da hipertensão no período gestacional. Reon Facema, 4, (3), 1231-1237.

Nagle, C., Skouteris, H., Hotchin, A., Bruce, L., Patterson, D, \& Teale, G. (2011). Continuity of midwifery care and gestacional weight gain in obese women: a randomised controlled trial. Bmc Public. Health, Austrália, 17 (11), 1-6.

Nascimento, A. A. J., Davoglio, R. S., Sousa, L. M., Figueiredo, A. C. M. G., Cruz, S. S. D. \& Filho, I. S. G.(2017). Hipertensão Materna e baixo peso ao nascer: Estudo caso-controle em dois municípios do semiárido Nordestino. Revista Baiana de Saúde Pública, 40 (1), 93-107.

Nóbrega, M. F., Santos, M. T. B. R., Davim, R. M. B., O, F. M., Alves, E. S. R. C. \& R, E. S. R. C. (2016). Perfil de gestante com síndrome hipertensiva em uma maternidade pública. Revista de Enfermagem, 5(10), 1805-1811.

Novo, J. L. V. G. \& Gianini, R. J.(2010). Mortalidade materna por eclampsia. Rev. Bras. Saúde Matern. Infant., 10 (2), $209-217$.

Peraçoli, J. C., Borges, V. T. M., Ramos, J. G. L., Cavalli, R. R., Costa, S. H. A. M., Oliveira, L. G., Souza, F. L. P., Korkes, H. A., Brum, I. R., Costa, M. L., Junior, M. D. C., Sass, N., Diniz, A. L. D., Prado, C.A. C. \& Filho, E.V.C. (2019). Pré-eclâmpsia/Eclampsia. Rev. Bras. Ginecol. Obstet., 41, $318-332$.

Pereira, G. T., Santos, A. A. P., Silva, J. M. O. \& Nagliate, P. C. (2017). Perfil Epidemiológico da Mortalidade Materna por Hipertensão: análise situacional de um estado nordestino entre 2004-2013. Rev. Fund. Care Online, 3(9), 653-658.

Rezende, G. P., Casagrande, L., Guida, J. P. S., Parpinelli, M. A., Surita, F. G. \& Costa M. L. (2020). Maternal and Perinatal Outcomes of Pregnancies Complicated by Chronic Hypertension Followed at a Referral Hospital. Rev. Bras. Ginecol. Obstet., 42 (5), $248-254$. 
Research, Society and Development, v. 10, n. 6, e1410615464, 2021

(CC BY 4.0) | ISSN 2525-3409 | DOI: http://dx.doi.org/10.33448/rsd-v10i6.15464

Rocha, E. S. S., Nunes, C. R, Cardoso, M. S. L. V. D., Marinho, E. A. R. O. \& Batista, R. S. (2016). Sistematização da Enfermagem na Doença Hipertensiva Específica da Gravidez em Adolescentes. Reinpec, 2 (2), 209-227.

Sampaio, A. F. S., Rocha, M. J. F. \& Leal, E. A. S. (2018). Gestação de alto risco: perfil clínico-epidemiológico das gestantes atendidas no serviço de pré-natal da maternidade pública de Rio Branco, Acre. Rev. Bras. Saúde Mater. Infant., 18 (3), 565-575.

Santos, L. V., Lara, M. O., Lima, R. C. R., Rocha, A. F., Rocha, E. M., Glória, J. C. R. \& Ribeiro, G. C. (2018). História ge stacional e características da assistência pré-natal de puérperas adolescentes e adultas em uma maternidade do interior de Minas Gerais, Brasil. Ciência Saúde Coletiva,23 (2), 617-625.

Santos, N. E.T., Leal, M. C., Oliveira, M. E., Zondonade, E, \& Gama, S. G. M. (2012). Concordância entre informações do Cartão da Gestante e da memória materna sobre assistência pré-natal. Cad. Saúde Pública, 28 (2), 256-266.

Silva, A. M., Rabito, L. B. F., Vaz, M. C., Santos, M. H. L., Vaz, L. C. \& Carvalho. E. R. (2017). O Enfermeiro Perante a Hipertensão Gestacional. Revista Iniciare, 2 (1), 22-26.

Silva, P. N., Oliveira, J. S., Santos, A. P. O. S. \& Vaz, M. D. T. (2017). Cuidados Pré-natais e puerperais às gestantes de um centro de saúde de Minas Gerais quanto ao risco de Pré-eclâmpsia: aspectos clínicos, nutricionais e terapêuticos. J. Health Biol. Sci, 5 (4), $346-351$.

Silva, M. P., Santos, Z. M. S. A., Nascimento, R. O. \& Fonteles, J. L. (2010). Avaliação das condutas de prevenção da Síndrome Hipertensiva Específica da gravidez entre adolescentes. Rev. Rene. Fortaleza, 11 (4), 57-65.

Soares, V. M. N., Souza, K. V., Freygang, T. C., Correa, V. \& Saito, M. R. (2009). Mortalidade materna por pré-eclâmpsia/Eclâmpsia em um estado do Sul do Brasil. Rev. Bras. Ginecol. Obstet., 31 (11). 566-573.

Solbiati, V. P., Oliveira, N. R. C., Teixeira, C. V. L. S. \& Gomes, R. J. (2018). Adesão ao Tratamento para Prevenir Agravos Relacionados a Hipertensão Arterial e ao Diabetes. Revista Brasileira de Obesidade, Nutrição e Emagrecimento, 12 (73), 629-633.

Sousa, M. G., Lopes, R. G., Rocha, M. L., Lippi, U. G., Costa, E. S. \& Santos, C. M. (2020). Epidemiologia Hipertensão arterial em gestantes. Einstein, 18. http://dx.doi.org/10.1744/einstein_journal/2020AO4682

Sousa, L. M. M. S., et al. (2017). Metodologia de revisão integrativa da literatura em enfermagem. https://www.researchgate.net/publication/321319742.

Sousa, M. G., Reginaldo, G. C. L., Rocha, M. L. T. L. F., Gazi, L. U., Costa, E. S. \& Santos, C. M. P. (2020). Epidemiologia da hipertensão arterial em gestantes. Einstein, 18, 1-7.

Spindola, T., Lima, G, L, S, \& Cavalcanti, R, L.(2013). A ocorrência de pré-eclâmpsia em mulheres primigestas acompanhadas no pré-natal de um Hospital Universitário. Journal Of Research Fundamental Care Online, 5 (3), 235-244.

Thuler, A. C. M. C., Wall, M. L., Benedet, D. C. F, Souza, R. R. K. \& Souza, M. A. R. (2018). Medidas Preventivas das Síndromes Hipertensivas da Gravidez na Atenção Primária. Rev. Enferm UFPE Online, 12 (4), 1060-1071.

Tuon, R, A., Ambrosano, G, M, B., Silva, S, M, C, V. \& Pereira, A, C. (2016). Impacto do monitoramento telefônico de gestantes na prevalência da prematuridade e análise dos fatores de risco associados em Piracicaba, São Paulo, Brasil. Cad. Saúde Pública, 32 (7),1-16.

Vettore, M. V., Dias, M., Domingues, R. M. S. R., Vettore, M. V. \& Leal, M.C.(2011). Cuidados pré-natais e avaliação do manejo da hipertensão arterial em gestantes do. Cad. Saúde Pública, 27 (5), 1021-1034. 